\title{
VHDL DESIGN AND HARDWARE REALIZATION OF HYBRIDARTIFICIAL INTELLIGENCE ARCHITECTURE
}

\author{
${ }^{1}$ Rajeswaran Nagalingam, ${ }^{2}$ Tenneti Madhu and ${ }^{3}$ Munagala Suryakalavathi \\ ${ }^{1}$ Department of ECE, SNS College of Technolgoy, Coimbatore, Tamilnadu, India \\ ${ }^{2}$ Principal,Swarnandhra Institute of Engg. and Tech., Narasapur Andhrapradesh, India \\ ${ }^{3}$ Department of EEE, Jawaharlal Nehru Technological University, Hyderabad Andhrapradesh, India
}

Received 2013-10-19; Revised 2013-11-11; Accepted 2014-02-23

\begin{abstract}
Evolutionary Algorithms (EA) use Genetic Algorithm (GA) in many optimization problems to efficiently compute the function value in less time. In this study the weight optimization of the Artificial Neural Network (ANN), using the Back Propagation Network (BPN), is tested and presented with GA. The combined architecture of Neuro-Genetic (Hybrid Artificial Intelligence) approach is proposed and simulated results are provided along with device Utilization, Simulation time, Timing analysis and power analysis by using very high speed integrated circuits Hardware Description Language (HDL).
\end{abstract}

Keywords: ANN, Evolutionary Algorithm, GA, Hybrid AI, VHDL

\section{INTRODUCTION}

Charles Darwin proposed the "Theory of Evolution" in the year 1858. The Evolutionary Algorithms (EA) are broadly classified into three categories. They are Evolutionary Strategies (ES), Evolutionary Programming (EP) and Genetic Algorithms (GA) (Palmes et al., 2005). Ingo Rechenberg and Hans-Paul Schwefel (1960s and early 1970s) solved complex engineering problems through artificial evolution strategies using optimization method. John Holland is the initiator for the development of Genetic algorithms in 1970s (Mitchell, 1998). Further, the motivation for the development of neural network technology stemmed from the desire to develop an artificial system that could perform "intelligent" tasks similar to those performed by the human brain. In 1943 the first neuron model was developed by the McCulloch and Pitts. Afterwards Evolutionary Computation (EC) and Neural Network technology impressed the interest of many researchers (Bose, 2007). A neural network is a powerful data modeling tool that is able to capture and represent the complex input/output relation-ships (Haykin, 1999). The development of back propagation algorithm in 1986 by Rumelhart and McClelland paved the way to research and development in the field of neuro Corresponding Author: Rajeswaran Nagalingam, Department of ECE, SNS College of Technolgoy, Coimbatore, Tamilnadu, India computation till now. Most of the other neural network structures represent models for "thinking" that are still being evolved in the laboratories. However, Genetic algorithms are a class of optimization procedures which are good at exploring a large and complex space in an intelligent way to find values close to the global optimum. Hence, they are well suited to the problem of training feed for-ward networks (Montana and Davis, 1989). The power and usefulness of artificial neural networks has been demonstrated in several applications including speech synthesis, diagnostic problems, medicine, business and finance, robotic control, signal processing, computer vision and many other problems that fall under the category of pattern recognition (Alsmadi et al., 2011).

The main advantage of evolutionary computation has inspired new resources for optimization problem solving, such as the optimal design of neural networks and fuzzy systems (Juang, 2004). Field Programmable Gate Array (FPGA) are becoming increasingly popular for prototyping and designing complex hardware systems. The structure of FPGA can be described as an array of blocks connected together via programmable interconnections (Blake et al., 1997; Sahin et al., 2006). The relatively low cost and easiness 
of implementation and reprogramming of FPGA's in comparison with the custom VLSI technology offer attractive features for the designer (Botros and AbdulArir, 1994; Ameur et al., 2012; Mahyuddin et al., 2009) Here in this study, the neuro-Genetic system is designed and simulated by using VHDL.

\section{NEURO-GENETIC APPROACH}

The traditional problem solving approach analyses the task and then derives a suitable algorithm. If successful, the result is immediately available. Neural networks can solve problems which are difficult to describe in analytical manner. But prior to usage, the network must be trained (Lange, 2005). A feed forward Back Propagation neural network is used to estimate the weights. The optimum selection of weights reduces the search-pace of the Genetic Algorithm (GA) to improve its performance (Kannaiah et al., 2011; Ditthakit and Chinnarasri, 2011). Figure 1, the evaluation function or fitness function is used to determine the fitness value of each candidate in the population. The proposed combined architecture will be helpful to solve the hardware implementation of the Hybrid AI.

\subsection{Training Algorithm of BPN}

It involves four steps (Sivanandam and Deepa, 2006)

Step 1: Initial weight selection

Step 2: Feed forward network

Step 3: Error calculation

Step 4: Updating of the weight and biases

The parameters are

$\mathrm{x}$ : Input training vector $\left(\mathrm{x}_{1}, \ldots \ldots \ldots \ldots \mathrm{x}_{\mathrm{n}}\right)$

$\mathrm{t}$ : Output target vector

$\Delta_{\mathrm{k}}$ : Error at output unit $\mathrm{y}_{\mathrm{k}}$

$\Delta_{\mathrm{j}}$ : Error at hidden unit $\mathrm{z}_{\mathrm{j}}$

$\alpha$ : Learning rate

$\mathrm{v}_{\mathrm{oj}}$ : Bias on hidden unit $\mathrm{j}$

$\mathrm{zj}$ : Hidden unit j

$\mathrm{w}_{\mathrm{ok}}$ : Bias on output unit $\mathrm{k}$

$\mathrm{y}_{\mathrm{k}}$ : Output unit k Equation (1):

$\mathrm{y}_{\mathrm{k}}=\mathrm{w}_{\mathrm{ok}} \sum_{\mathrm{j}=1}^{\mathrm{n}} \mathrm{z}_{\mathrm{j}} \mathrm{w}_{\mathrm{jk}}$

\subsection{Procedure for Genetic Algorithm}

The important parameters used in genetic algorithm are crossover rate, mutation rate, population size, selection, encoding and crossover and mutation type.

The algorithm is as follows:
Step 1: Initial- Random selection of population

Step 2: Select parents from population

Step 3: Perform crossover on parents creating an offspring

Step 4: Mutation operation for the best Individuals (offspring)

Step 5: Determine the fitness

Step 6: Repeat the process for the selection of the best individual and discard the worst

\subsection{Pseudo Code for Proposed Design}

va0,va1,va2: In integer range 1 to 15 ; std_logic_vector(7 downto 0);

$\mathrm{y}$ : Inout integer range 1 to 15$)$;

std_logic_vector(7 downto 0));

signal za,zb,zc: Integer range 1 to 15 ;

component lfsr12bit is

clk, rst : In std_logic;

Ifsr_reg : Inout std_logic_vector(11 downto 0));

end component; GA

Rand: In std_logic_vector(3 downto 0$)$;

Wheel: In std_logic_vector(3 downto 0$)$;

GA_STORE1: Inout std_logic_vector (5 downto 0);

GA_STORE2: Inout std_logic_vector (5 downto 0);

----processing of 1st layer -- identity activation function

for hidden layer nodes---for number in 1 to 50 loop

$$
\text { if }(\mathrm{t} /=\mathrm{y}) \text { then }
$$

$\mathrm{za} 0<=\left(\left(\mathrm{xa}^{*} \mathrm{va} 0\right)+(\mathrm{xb} * \mathrm{vb} 0)+(\mathrm{xc} * \mathrm{vc} 0)+\mathrm{v} 0\right)$;

$\mathrm{zb} 0<=\left(\left(\mathrm{xa}^{*} \mathrm{va} 1\right)+\left(\mathrm{xb}^{*} \mathrm{vb} 1\right)+(\mathrm{xc} * \mathrm{vc} 1)+\mathrm{v} 1\right) ;$

--identity activation function for hidden layer nodes if (clk'event and clk = ' 1 ') then

if $($ reset $=$ ' 1 ') then

$\mathrm{za}<=1$;

-- calculation of error information

if(clk'event and clk =' 1 ') then

if $($ reset $=$ ' 1 ') then

err_hid_za $<=1$;

if(clk'event and clk =' 1 ') then

if $($ reset $=$ ' 1 ') then

va0_new $<=1$;

---calculation of weight compensation value

if(clk'event and clk =' 1 ') then

if $\left(\right.$ reset $={ }^{\prime} 1$ ') then

del_w0<= 1 ;

---calculation of new weight if $\left(\right.$ clk'event and clk $={ }^{\prime} 1$ ') $)$ then

if $($ reset $=$ ' 1 ') then

end process; 


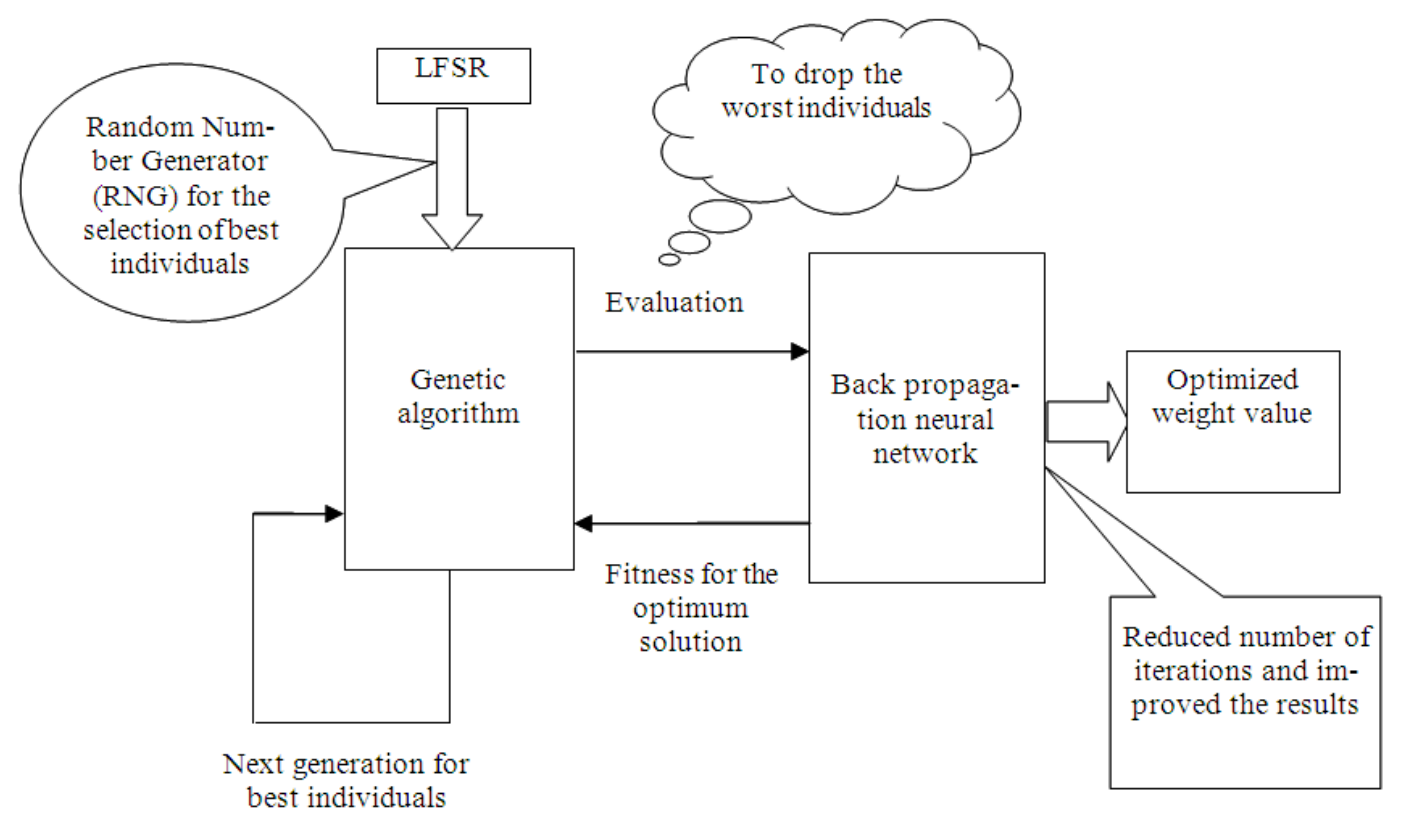

Fig.1. Proposed neuro-genetic approach

\section{RESULTS}

GA with BPN is tested by using the hardware unit xc3s500e-4-pq208. The simulation result, device utilization and timing summary are tabulated in Table 1 to 3 respectively. The total number of Pins in the device are 208 and used pins in the proposed design are 49 only. Net skew is the difference between the minimum and maximum routing only delays for the net. Clock skew is the difference between the minimum and maximum path delays which include logic delays. The obtained clock report is presented in Fig. 4. Here the combined architecture (Fig. 2) used the roulette wheel selection method for fitness calculations. Based on the application, different methods can be used for fitness calculations. The total memory usage is 167380 kilobytes. The Total time to complete the simulation is $6.189 \mathrm{~ns}(4.567 \mathrm{~ns}$ logic, 1.622 ns route) $(73.8 \%$ logic, $26.2 \%$ route). Total REAL time to Xst completion: $11.00 \mathrm{sec}$ and Total CPU time to Xst completion: $11.48 \mathrm{sec}$. The output of the neuro-genetic is given in Fig. 3. The selected device consumed voltage, thermal and power information's are given in the screen shots (Fig. 5-7). It is evident that the utilization of hardware and efficiency to reduce power consumption in various factors of the proposed design are achieved.

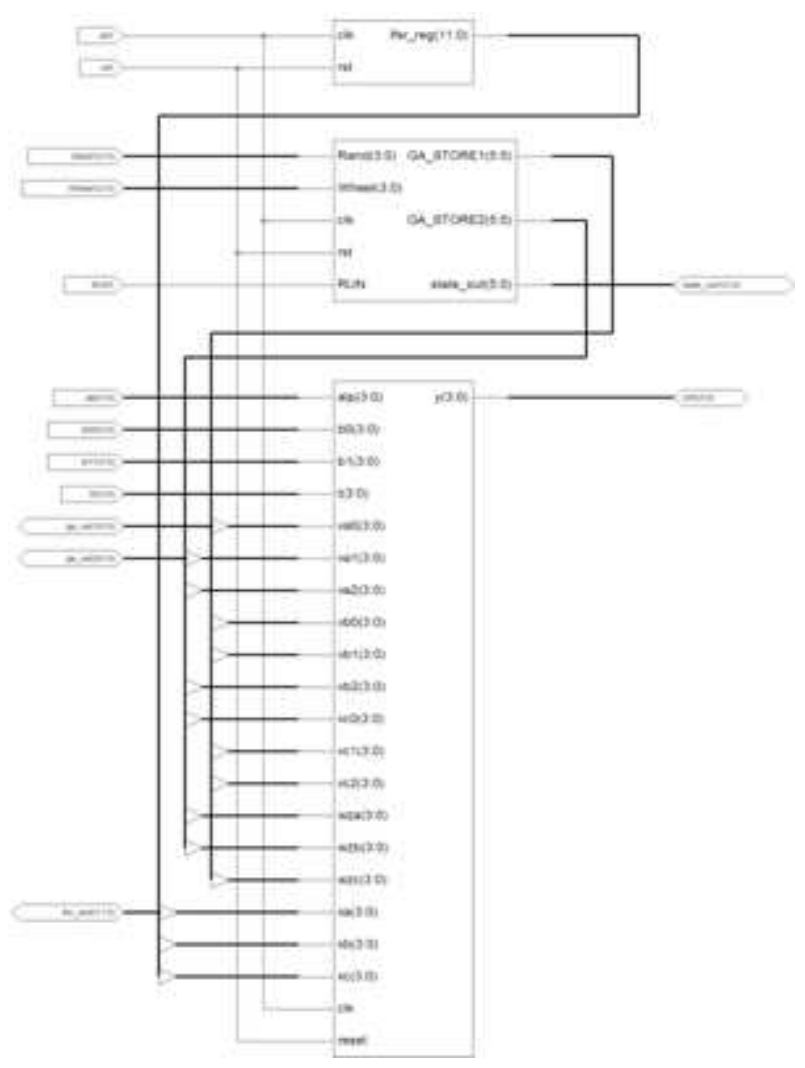

Fig. 2. FPGA implementation of Hybrid AI 
Rajeswaran Nagalingam et al. / American Journal of Applied Sciences 11 (5): 782-788, 2014

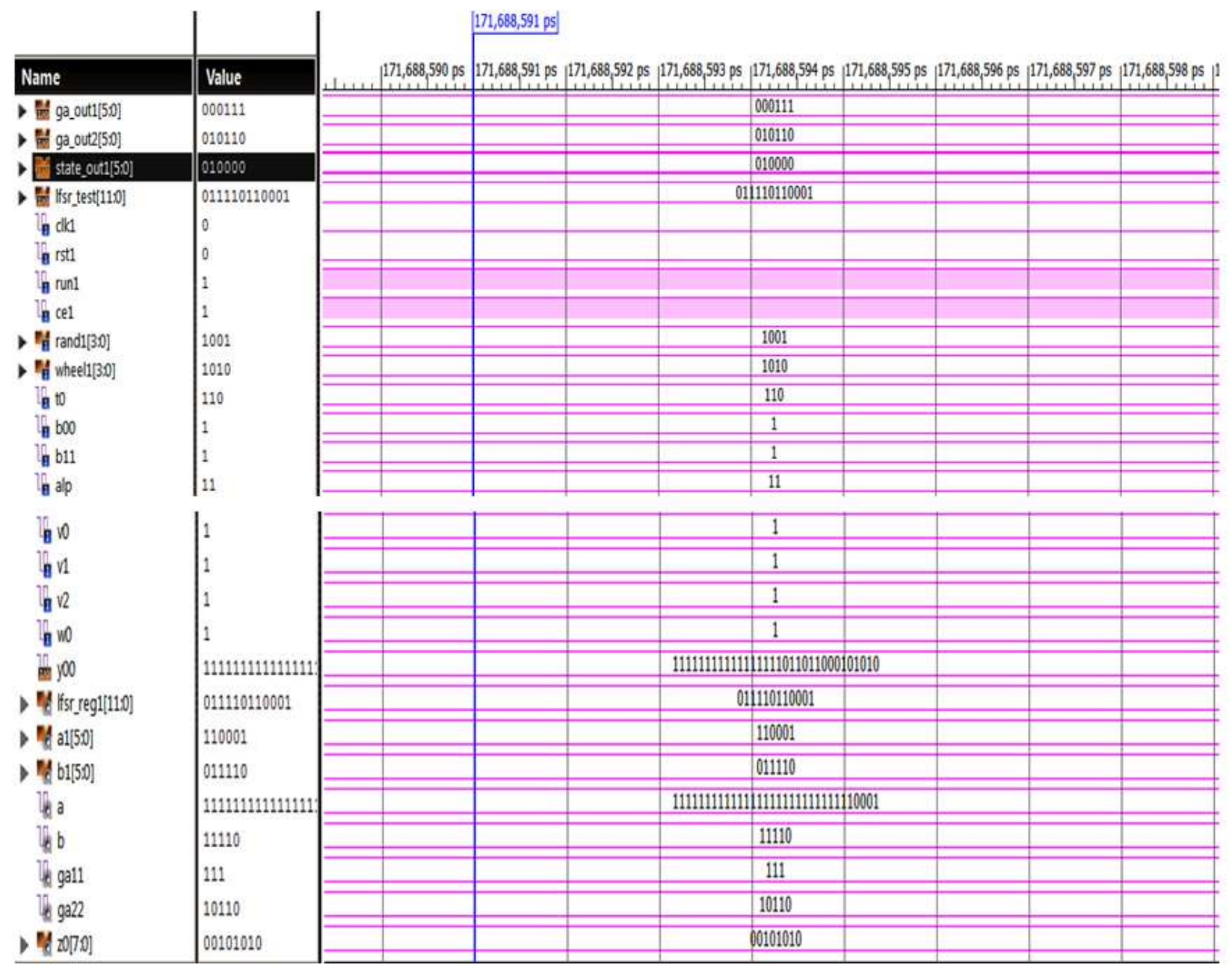

Fig. 3. Output waveform of proposed design

\begin{tabular}{l|l|r|r|r|r|} 
Clock Net & Resource & Locked & Fanout & Net Skew(ns) & Max Delay(ns) \\
\hline state_out1_1_OBUF & BUFGMUX_X1Y11 & No & 11 & 0.025 & 0.157 \\
\hline clk1_BUFGP & BUFGMUX_X2Y10 & No & 34 & 0.029 & 0.153 \\
\hline x2/ov2 & Local & & 6 & 0.071 & 1.297 \\
\hline x2/ov4 & Local & & 15 & 0.758 & 2.559 \\
\hline x2/ov1 & Local & & 6 & 0.045 & 2.000 \\
\hline x3/y0_not0001 & Local & & 16 & 0.488 & 2.408 \\
\hline x2/ov3 & Local & & 5 & 0.123 & 2.010 \\
\hline
\end{tabular}

Fig. 4. Clock report 
Rajeswaran Nagalingam et al. / American Journal of Applied Sciences 11 (5): 782-788, 2014

\begin{tabular}{|l|l|l|l|l|l|}
\hline Name & Power $\left(W^{\prime}\right)$ & Range & Vollage & $\operatorname{Icc}(\mathrm{A})$ & $\operatorname{Iccq}(\mathrm{A})$ \\
\hline Vocint & 0.031 & 1.14101 .26 & 1.20 & 0.000 & 0.026 \\
\hline Vecaux & 0.045 & & 2.5 & 0.000 & 0.018 \\
\hline Veco25 & 0.005 & & 2.5 & 0.000 & 0.002 \\
\hline
\end{tabular}

Fig. 5. Voltage source information

\begin{tabular}{|c|c|c|}
\hline Name & Value & Range \\
\hline Ambient Temp (degrees C) & 25.0 & -20.0 to 85.0 \\
\hline ThetalA (degrees $\mathrm{C} / \mathrm{W}$ ) & 36 & \\
\hline Use custom ThetalA? & Yes & \\
\hline Custom ThetalA (degrees $\mathrm{C} / \mathrm{W}$ ) & & 0.0 to 20.0 \\
\hline Airflow (LFM) & NA & 0 to 750 \\
\hline Max Ambient (degrees C) & 82.1 & \\
\hline Junction Temp (degrees C) & 27.9 & \\
\hline
\end{tabular}

Fig. 6. Thermal information

\begin{tabular}{|l|l|l|}
\hline Name & Value & Range \\
\hline FF Toggle Rate [\%] & 12.5 & 0.0 to 100.0 \\
\hline I/O Toggle Rate [\%] & 12.5 & 0.0 to 100.0 \\
\hline Output Load (pF] & 5.0 & 0.0 to 1000000.0 \\
\hline I/O Enable Rate [\%] & 100.0 & 0.0 to 100.0 \\
\hline BRAM Write Rate [\%] & 50.0 & 0.0 to 100.0 \\
\hline BRAM Enable Rate [\%] & 25.0 & 0.0 to 100.0 \\
\hline DSP Toggle Rate [\%] & 12.5 & 0.0 to 100.0 \\
\hline & & \\
\hline Part & $3 s 500$ epq208-4 & \\
\hline Package & pq208 & \\
\hline Grade & Commercial V \\
\hline Process & Typical & \\
\hline
\end{tabular}

Fig. 7. Power analyzer summary

Table 1. Device utilization summary

\begin{tabular}{lrrr}
\hline Logic utilization & Used & Available & Utilization (\%) \\
\hline Total number slice registers & 119 & 9312 & 1 \\
Number used as flip flops & 43 & & \\
Number used as latches & 76 & 9312 & 1 \\
Number of 4 input LUT's & 96 & 4656 & 2 \\
Number of occupied slices & 107 & 158 & 31 \\
Number of bonded IOB's & 49 & 20 & 60 \\
Number of mulT18X18SIOs & 12 & 24 & 8 \\
Number of BUFGMUXs & 2 & & \\
\hline
\end{tabular}


Table 2. Timing summary

\begin{tabular}{lc}
\hline Parameters & Time (ns) \\
\hline Minimum period & 4.552 \\
Minimum input arrival time before clock & 5.711 \\
Maximum output required time after clock & 6.189 \\
Maximum combinational path delay & No path found \\
\hline
\end{tabular}

Table 3. Simulation parameters

\begin{tabular}{|c|c|c|}
\hline Parameters & Name of the signals & Value \\
\hline RUN1 & Processing signal & 1 \\
\hline clk1 & Input & Rising edge \\
\hline rst1 & Input & 1 \\
\hline ce1 & Processing signal & 1 \\
\hline Rand1 & Fitness & 4 Bits \\
\hline Wheel1 & Fitness & 4 Bits \\
\hline GA_STORE1 & Inout & 6 Bits \\
\hline GA_STORE2 & Inout & 6 Bits \\
\hline state_out & Inout & 6 Bits \\
\hline t0 & Target output & Integer range 1 to 15 \\
\hline b00 & Bias 1 & Integer range 1 to 15 \\
\hline b11 & Bias2 & Integer range 1 to 15 \\
\hline alp & Learning rate & Integer range 1 to 15 \\
\hline $\mathrm{v} 0$ & Weight of input layer & Integer range 1 to 15 \\
\hline v1 & Weight of input layer & Integer range 1 to 15 \\
\hline v2 & Weight of input layer & Integer range 1 to 15 \\
\hline w0 & Weight of output layer & Integer range 1 to 15 \\
\hline lfsr & Linear feedback shift register & 12 Bits \\
\hline y00 & Actual output & Integer range 1 to 15 \\
\hline
\end{tabular}

\section{CONCLUSION}

The proposed method of neuro-genetic hardware architecture design is one of the successful implementation methods of Hybrid AI in FPGA. The device utilization, clock report, power summary, voltage source information, thermal Information and power analyzers show that the proposed method is achieving the required speed and efficiency. In future the proposed method will be used for different real time applications.

\section{REFERENCES}

Alsmadi, M.K., K.B. Omar and S.A.M. Noah, 2011. Fish classification based on robust features extraction from color signature using back-propagation classifier. J. Comput. Sci., 7: 52-58. DOI: 10.3844/jcssp.2011.52.58

Ameur, M.S.B., A. Sakly and A. Mtibaa, 2012. Implementation of genetic algorithms using FPGA technology. Proceedings of the Annual FPGA Conference, Sep. 4-4, New York, DOI: $10.1145 / 2451636.2451639$
Blake, J.J., L.P. Maguire, T.M. McGinnity and L.J. MCDaid, 1997. Using xilinx FPGAs to implement neural networks and fuzzy systems. Proceedings of the Colloquium on Neural and Fuzzy Systems: Design, Hardware and Applications, May 9-9, IEEE Xplore Press, London, pp: 1-4. DOI: 10.1049/ic:19970730

Bose, B.K., 2007. Neural network applications in power electronics and motor drives-an introduction and perspective. IEEE Trans. Indust. Electron., 54: 1433. DOI: 10.1109/TIE.2006.888683

Botros, N.M. and M. Abdul-Arir, 1994. Hardware implementation of an artificial neural network using. Field Programmable Gate Arrays (FPGA's). IEEE Trans. Indust. Electron., 41: 665-667. DOI: 10.1109/41.334585

Ditthakit, P. and C. Chinnarasri, 2011. Estimation of pan evaporation coefficient using neuro-genetic approach. Am. J. Environ. Sci., 7: 397-401. DOI: 10.3844/ajessp.2011.397.401

Haykin, S.S., 1999. Neural Networks: A Comprehensive foundation. 2th Edn., Prentice Hall International, Upper Saddle River, ISBN-10: 0139083855. pp: 842. 
Juang, C.F., 2004. A hybrid of genetic algorithm and particle swarm optimization for recurrent network design, IEEE Trans. Syst, Man Cybern. B Cybern., 34: 997-1006. DOI: 10.1109/TSMCB.2003.818557

Kannaiah, S.K., J. Thangavel and D.P. Kothari, 2011. A genetic algorithm based multi objective service restoration in distribution systems. J. Comput. Sci., 7: 448-453. DOI: 10.3844/jcssp.2011.448.453

Lange, R., 2005. Design of a generic neural network fpga-implementation. Proceedings of the CHEMNITZ University of Technology, Faculty of Electrical Engineering and Information Technology, Professorship of Circuit and Systems Design, (SD '05), pp: 1-143.

Mahyuddin, M.N., C.Z. Wei and M.R. Arshad, 2009. Neuro-fuzzy algorithm implemented in Altera's FPGA for mobile robot's obstacle avoidance mission. Proceedings of the IEEE Region 10th Conference TENCON, Jan. 23-26, IEEE Xplore Press, Singapore, pp: 1-6. DOI: 10.1109/TENCON.2009.5396012
Mitchell, M., 1998. Introduction to Genetic Algorithm. 1st Edn., MIT Press, Cambridge, Mass, ISBN-10: 0262631857. pp: 209.

Montana, D.J. and L. Davis, 1989. Training feedforward neural networks using genetic algorithms. Proceedings of the 11th International Joint Conference on Artificial Intelligence (IJCAI '89), San Francisco, pp:762-767.

Palmes, P.P., T. Hayasaka and S. Usui 2005. Mutationbased genetic neural network. IEEE Trans. Neural Netw.,16: 587-600.

DOI: 10.1109/TNN.2005.844858

Sahin, S., Y. Becerikli and S. Yazici, 2006. Neural network implementation in hardware using FPGAs. Proceedings of the 13th International Conference, Oct. 3-6, Hong Kong, China, pp: 1105-1112. DOI: 10.1007/11893295_122

Sivanandam, S.N. and S.N. Deepa, 2006. Introduction to Neural Networks using Matlab 6.0. 1st Edn., Tata McGraw-Hill Education, New Delhi, ISBN-10: 0070591121. pp: 656. 